\title{
Role of bisphosphonates in treatment of osteoporosis in community. atypical periprosthetic sub trochanteric femur fractures during bisphosphonates therapy, a case report
}

\begin{abstract}
Bisphosphonates are used to treat postmenopausal and glucocorticoid-induced osteoporosis, Paget's disease of bone, and malignant hypercalcemia. They have been related to atypical femur fractures. We describe an 83-year-old woman on longterm bisphosphonates presenting with an atypical femur fracture. She underwent a surgical procedure, and a few months later, she presented with a nonunion of the periprosthetic femoral fracture. She underwent an additional surgical procedure and discontinued bisphosphonate treatment. As more atypical femur fractures associated with bisphosphonate use are being reported in the literature, the risk of atypical femur fracture with bisphosphonate use should not be ignored.
\end{abstract}

Keywords: fractures, bisphosphonates, postmenopausal, glucocorticoid, osteoporosis, femur fractures, pyrophosphate, cortical spike, radiographs, low energy, chronic obstructive pulmonary disease, thickening
Volume I Issue 2 - 2017

\section{Muhammad Baig}

Kerry General Hospital, Ireland

Correspondence: Muhammad Baig, Kerry General Hospital, Ireland, Email nouman 142@gmail.com

Received: July 27, 2017 | Published: September 20, 2017

\section{Introduction}

Osteoporosis is an important health challenge, and bisphosphonates provide the most benefit with the least cost. Bisphosphonates are drugs that inhibit mineralization or resorption of the bone by blocking the action of osteoclasts. Bisphosphonates are enzyme-resistant analogs of pyrophosphate, which normally inhibits mineralization in the bone. They reduce bone turnover by inhibiting osteoclast recruitment and promoting osteoclast apoptosis. The medical literature reports bisphosphonates are a potential cause of atypical femur fractures. Atypical fractures are characterized by transverse and noncomminuted fracture patterns in the subtrochanteric or femoral shaft regions with a medial cortical spike at the fracture area. Other features include prodromal pain and generalized thickening of the femoral cortices on radiographs. ${ }^{1,2} \mathrm{We}$ observed most of these features in the present case.

\section{Case presentation}

An 83-year-old woman presented to our hospital with low-energy trauma resulting in pain on the right thigh causing an inability to bear weight. She has a history of chronic obstructive pulmonary disease, type 2 diabetes mellitus, gastro esophageal reflux disease, and osteoporosis. She had an intertrochanteric fracture in the right neck of her femur in 2010 that was internally fixed using a dynamic hip screw (DHS) with a two-hole plate (Figure 1) (Figure 2). She was started on alendronate therapy in 2011 for osteoporosis. She had a fracture in the left distal radius in 2013 and underwent manipulation under anesthesia and Kirschner wire fixation. She experienced low-energy trauma in January 2015 and presented with right thigh pain and was unable to bear weight.

Radiological evaluation of the right femur and pelvis showed an atypical subtrochanteric femur fracture of the right leg (Figure 3). She underwent a surgical operation the next day, and a two-hole plate was removed without removing the lag screw and was replaced using a DHS with an eight-hole plate (Figure 4). She started physiotherapy, and within few weeks, she was walking with a walking frame. She received regular follow-up radiological examinations. Her alendronate was discontinued, and she was started on teriparatide injections. Teriparatide is a synthetic parathyroid hormone that exists naturally in the body. She was undergoing this treatment when, in April 2015, she had another low-energy fall and was brought to the hospital with pain in the right thigh and was unable to bear weight. On radiologic examination, she had DHS implant failure and nonunion of the subtrochanteric fracture (Figure 5). She underwent an additional surgical procedure, and the DHS was removed. A long cephalomedullary nail was inserted (Figure 6). She started physiotherapy again. The patient is receiving regular outpatient follow-up and radiologic examinations. She is walking with a walking frame and continues to receive teriparatide injections.

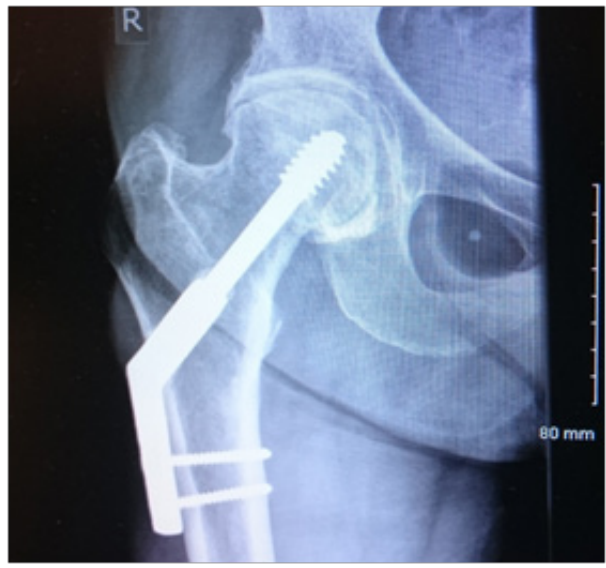

Figure I Dynamic hip screw with two-hole plate for neck of femur fracture. 


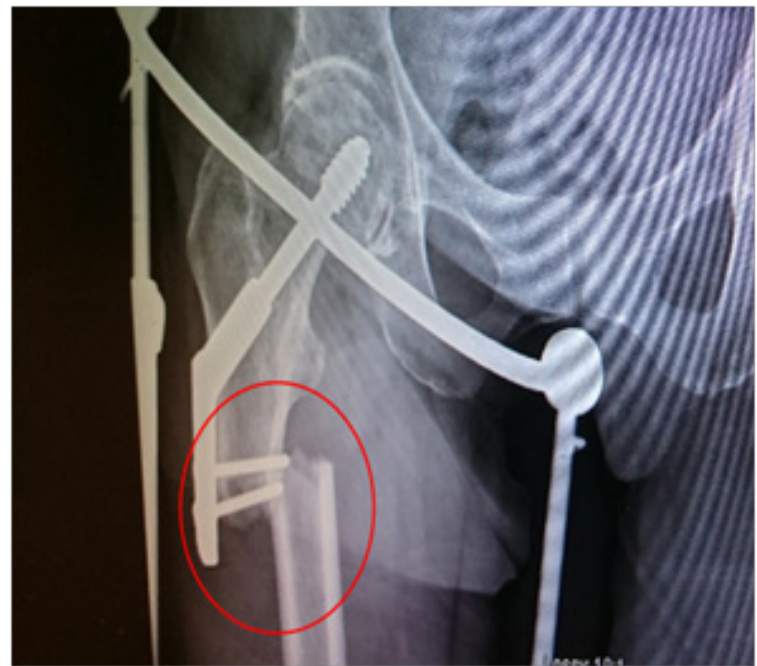

Figure 2 Subtrochanteric femur fracture (denoted by a red circle).

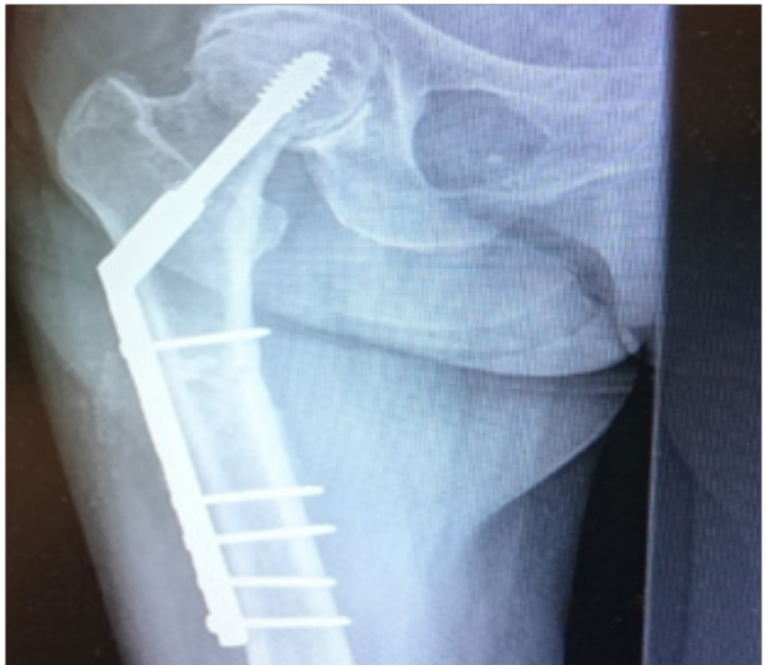

Figure 3 Conversion to dynamic hip screw with 10-hole plate.

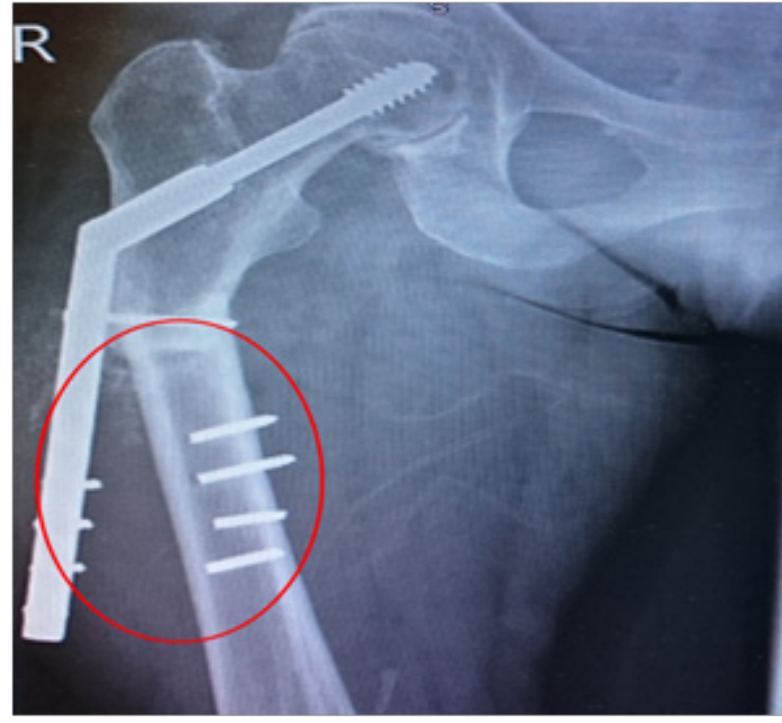

Figure 4 Periprosthetic fracture (denoted by a red circle).

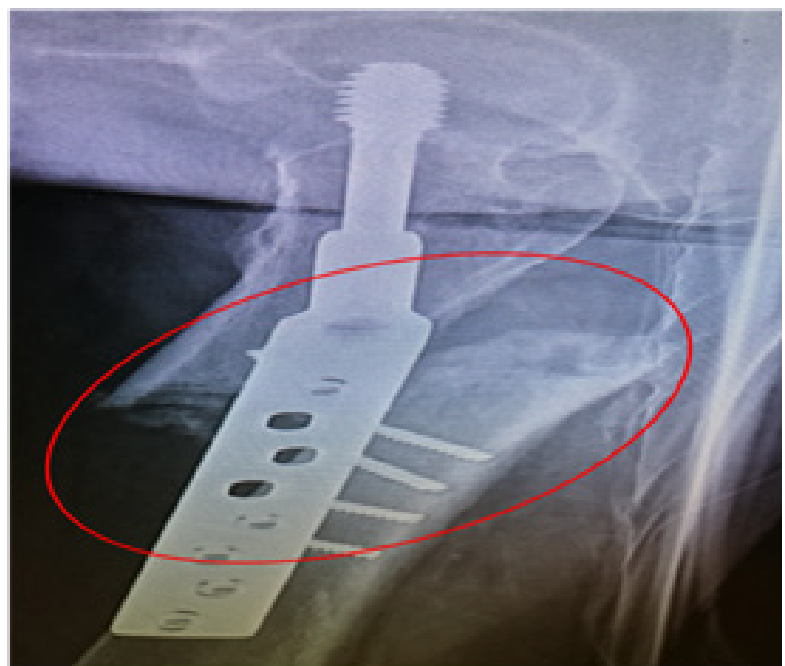

Figure $\mathbf{5}$ Lateral view of periprosthetic fracture (denoted by a red circle).

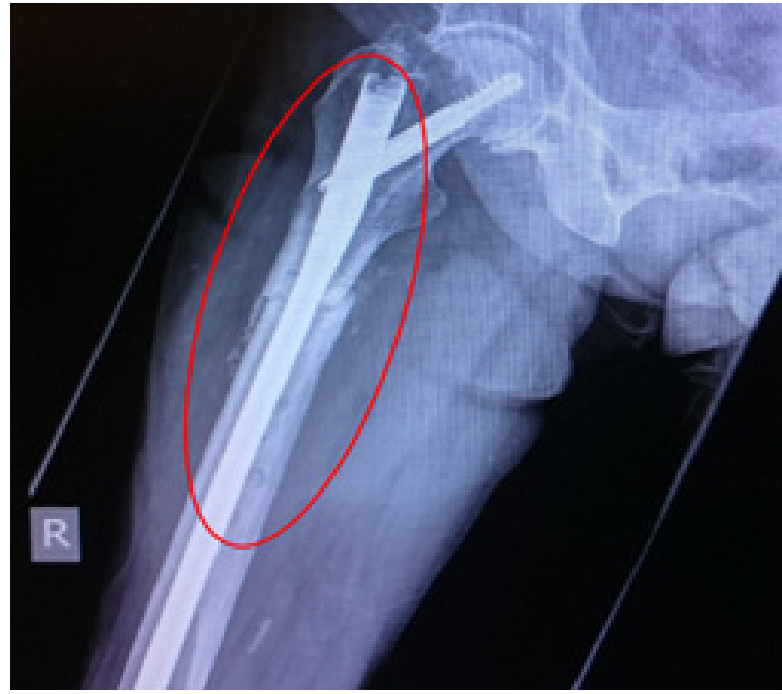

Figure 6 Cephalomedullary nailing of fracture (denoted by a red circle).

\section{Discussion}

Bisphosphonates are the primary pharmacological agents used to treat osteoclast-mediated bone loss due to osteoporosis, Paget's disease of bone, malignancies that metastasize to bone, multiple myeloma, and hypercalcemia of malignancy. In addition to currently approved uses, bisphosphonates are commonly prescribed for prevention and treatment of a variety of other skeletal conditions such as low bone density and osteogenesis imperfecta. ${ }^{3,4}$ Since their introduction into clinical practice, bisphosphonates have transformed the clinical care of an array of skeletal disorders characterized by excessive osteoclast-mediated bone resorption. Bisphosphates act as strong inhibitors of bone resorption by suppressing the activity of osteoclasts, and although this improves osteoporotic conditions, it also reduces overall bone turnover. ${ }^{5}$ Prolonged bisphosphonateinduced impairment of bone remodeling may cause an accumulation of microfractures and weakening of bone. ${ }^{6-7}$ Atypical femur fractures are associated with duration of bisphosphonate therapy. The risk of atypical femur fracture had previously been associated with the use of glucocorticoids and proton-pump inhibitors. ${ }^{8}$ Continued use of 
bisphosphonate therapy beyond a treatment period of three to five years should be re-evaluated annually based on pertinent medical history and medication regimen, starting and ending with bone density and fracture history. Consideration should be given to stopping bisphosphonate therapy, at least temporarily, in those patients who are assessed to be at low or low-to-moderate risk, defined as: (1) no incident fractures, (2) T-score $>-2.0$, and (3) no other major risk factors after a 3-to5-year therapeutic period. ${ }^{8}$ Bisphosphonates are effective in the primary prevention of symptomatic fragility fractures during the first few years of use in female patients with osteoporosis. ${ }^{9}$

\section{Conclusion}

The use of bisphosphonates is beneficial for osteoporosis, but after a certain period of time if its used in a prolonged way it can increase the risk of atypical femoral shaft fractures. Therefore, it is important for both the clinicians and patients to use bisphosphonates for proper indications keeping in mind the duration of its use as well.

\section{Acknowledgements}

None.

\section{Conflict of interest}

The author declares no conflict of interest.

\section{References}

1. Neviaser AS, Lane JM, Lenart BA, et al. Low-energy femoral shaft fractures associated with alendronate use. J Orthop Trauma. 2008;22(5):346-350
2. Schneider JP. Should bisphosphonates be continued indefinitely? An unusual fracture in a healthy woman on long-term alendronate. Geriatrics. 2006;61(1):31-33.

3. Drake MT, Clarke BL, Khosla S. Bisphosphonates: mechanism of action and role in clinical practice. Mayo Clin Proc. 2008;83:1032-1045.

4. Odvina CV, Zerwekh JE, Rao DS, et al. Severely suppressed bone turnover: a potential complication of alendronate therapy. $J$ Clin Endocrinol Metab. 2005;90(3):1294-1301.

5. Allen MR, Burr DB. Three years of alendronate treatment results in similar levels of vertebral micro damage as after one year of treatment. $J$ Bone Miner Res. 2007;22(11):1759-1765.

6. Black DM, Schwartz AV, Ensrud KE, et al. Effects of continuing or stopping alendronate after 5 years of treatment: The Fracture Intervention Trial Long-term Extension (FLEX): a randomized trial. JAMA. 2006;296(24):2927-2938.

7. Baig MN, Yousaf I, Galbraith JG, et al. Absorbable Polydioxanone (PDS) suture provides fewer wound complications than polyester (ethibond) suture in acute Tendo-Achilles rupture repair. Ir Med J. 2017;110(5):566.

8. Schilcher J, Koeppen V, Aspenberg P, et al. Risk of atypical femoral fracture during and after bisphosphonate use: Full report of a nationwide study. Acta Orthopaedica. 2015;86(1):100-107.

9. Girgis CM, Seibel MJ. Atypical femur fractures: a review of the evidence and its implication to clinical practice. Ther Adv Musculoskeleta Dis. 2011;3(6):301-314. 